\title{
Effects of Client Violence on Therapeutic Self-disclosure in Correctional and Non-correctional Settings
}

\author{
Robert Vavrinak, Kirk Lunnen \\ Westminster College, New Wilmington, USA
}

\begin{abstract}
Treating clients with a potential for violence may affect therapist behaviors during psychotherapy. It may affect therapist's use of a type of self-disclosure known as self-involving statements. This was examined by sending the CATS (Client's Assessment of Therapist Self-reference) survey to 144 psychologists within the US (United States) with age $53(S D=10)$ and with 23 years of experience $(S D=11)$. The CATS survey asked questions such as how often a person expresses either statements of disdain for his or her actions or happiness for his or her progress. This survey was administered to 22 individuals practicing psychotherapeutic techniques within a prison setting in response to a perceived increase in the amount of violence that occurs within these types of institutions and 122 worked in other fields, such as community hospitals and schools. The results of the study show that there is a difference in reported use of both positive and negative self-involving statements, with the correctional population group using fewer self-involving statements overall and having different motives for use when compared to a previously documented population of psychotherapist. This study also shows that anxiety may be the cause of these differences with roughly $2 / 3$ of the psychotherapists reporting that throughout their career they have been threatened with bodily harm by a client, and about half reporting that there was a significant need to activate emergency responses. Therapists in the non-correctional setting also tended to endorse more strengthening alliances and sharing or being open with the client rather than therapists in the correctional setting.
\end{abstract}

Keywords: self-involving statements, disclosure, client violence

\section{Introduction}

Therapist anxiety can affect the alliance a therapist has with a client and the methods the therapist would use to treat the client. One therapeutic technique that may be affected would be the use of therapist self-disclosure. Therapist self-disclosure is a broad field with sub-fields including but not limited to self-referent and self-involving statements. The current study focuses on the use of self-involving statements that the therapists stated evaluations of a client's progress. Self-involving statements differ from self-referent statements, because self-referent statements are the use of facts of events within the therapist's daily life used to convey personal data to a client. Client violence was examined as a possible source of therapist anxiety. Using client violence as a marker of potential therapist anxiety stems from the ability to count the number of occurrences of threats of assaultive behavior as seen in Guy, Brown, and Polestra (1992). This study analyzes how an increase in client violence can affect the type of self-involving statements (positive or negative) and the

Robert Vavrinak, Department of Psychology, Westminster College.

Kirk Lunnen, Ph.D., Department of Psychology, Westminster College. 
number of self-involving statements that the therapist uses.

\section{Self-disclosure/Self-involving Statements}

Therapist self-disclosure is any statement that a therapist makes to involve some aspects of the therapist's personal history, thoughts, or actions. There are some minor differences between self-involving statements and other types of therapist self-disclosure. McCarthy and Betz (1978) defined the self-involving statement as a here-and-now evaluation of the clinician's feelings of the client, whereas other forms of disclosure are more self-referent. A self-involving statement would be when the therapist states approval or disapproval of a client's actions. As opposed to a self-referent statement would be where a therapist gives information about one's personal life, such as experiences pertaining to the issue in the therapist's life. McCarthy and Betz (1978) found that clinicians who used self-involving statements were found to be more expert and trustworthy than clinicians who used self-referent statements. Reynolds and Fischer (1983) also found that there was no significant distinction in the ratings of trustworthiness and expertise between clinicians who used positive vs. negative self-involving statements. Another finding of Reynolds and Fischer (1983) was that clinicians who used more self-involving statements focused most of their therapy session on the client, whereas clinicians who used more self-referent statements asked more questions about themselves and spent less time talking about the client. B. Anderson and W. Andersen (1985) compared the levels of perceived alliance between positive and negative self-involving statements finding that therapists using positive self-involving statements were rated by the clients as being more appropriate attractive and trustworthy, and that the clients were more willing to see the therapist again than in the negative condition. Dowd and Barato (1982) showed that therapists who used more self-disclosure and self-involving statements were looked upon more favorably by clients. The study also found that these therapists were frequently rated as having a higher preference rating. These results showed that self-involving statements could play a major role on the alliance between a therapist and a client. The study by Barnett and Lunnen (2008) was the source of the modified version of the CATS (Client's Assessment of Therapist Self-reference) survey that was used in this study, they made a derivation of the therapist self-involving statement questionnaire by adding questions on frequency of the use of each statement and reasoning for the use of these statements. This was further modified to add questions about therapist anxiety and the fears of client violence.

\section{Therapy With Violent Clients}

Some insecure clients with severe mental disorders can be a major issue for psychotherapists. A meta-analysis by Hudson-Allez (2006) revealed that nearly 1/4 of psychotherapists were the victim of stocking by former clients as was found to be a significant difference compared to the normal everyday population, which was found to be a little more than $1 / 10$ of people. This study described stalking as "a constellation of intrusive and unwanted behaviors that one person inflicts on another"; it also stated that "such behaviors may involve persistent communications (letters, telephone calls, emails, and texts); watching, following, or approaching the individual; or ordering goods or cancelling services obstensively on the victim's behalf” (Hudson-Allez, 2006, p. 249). Hudson-Allez went on to talk about how these clients who stalked may have developed an insecure attachment, stating that clients with an insecure attachment style may suffer from an inability to handle a short-term professional relationship with therapists. Hudson-Allez described the tendency of stalking as "a protest reaction designed to regain contact with the attachment figure may become manifest in persistent and needy stalking behaviors”. Hudson-Allez also stated that there may 
be some sexual attractions or intimacy issues within a client. This insecurity may also manifest itself in another manner, such as violence, especially among clients who have a history of using aggressive tendencies toward psychotherapists.

The threat and use of violence by clients has been examined in a meta-analysis by Glanci and Saini (2005, p. 229) who found that in most situations, aggressive actions are usually directed at another person, with over half of anger episodes, targeting a loved one or an acquaintance. This is significant because most psychotherapists aim to develop strong therapeutic alliances with clients, making them possible targets for these aggressive actions. Glanci and Saini (2005, p. 230) reported that the tendency to treat clients with anger problems has rapidly increased. The therapists in their study reported treating angry clients as often as they treated clients with anxiety issues. This article goes into describing many methods to clinically deal with violent clients in a therapeutic manner. Guy, Brown, and Polestra (1992) studied the use and threat of violence within a clinical setting and the likelihood of people to follow through on that threat. They discovered that about $40 \%$ of the psychologists polled had been physically assaulted by a patient. Descriptions of these incidents revealed that these attacks "ranged in severity from the use of feet, fists, and pencils to the use of guns, knives, and ropes” (Guy, Brown, \& Polestra, 1992, p. 423). Also, these psychotherapists had been attacked from one to 20 times per clinician with a total number of 301 attacks reported. Guy et al. (1992) also reported that almost $1 / 2$ of all clinicians had been threatened with physical violence; they also found that $52 \%$ of the psychologists who had reported being threatened had actually been attacked. These results showed that client violence should be a major concern for psychotherapists, and those attacks can happen on any clinician, especially when dealing with clients with a violent past.

There are plenty of training methods used in treating violent clients. Hunter (1989) asked questions about the frequency of use of restraining techniques on juvenile clients with violent histories and found that around $65 \%$ of the clinicians reported using physical restraint on juvenile clients within recent weeks. In a related study, Sharkin and Gelso (1993) used the trait anger scale to assess how angry each individual patient and trainee therapist were before treatment. They found that trainee therapists who were more prone to anger were more likely to express anger toward a client who also had a tendency to be prone to anger. This can damage the therapeutic alliance between the clinician and the client they are treating, which could hinder progress within clients by the perception that their clinician is uncomfortable around them. Another possible outcome of this is a high level of therapist anxiety which may also lower the success of the therapy.

Anxiety can affect the relationship that a psychotherapist has with his or her client. In a meta-analysis by Harris (1999), he examined how the alliance between the therapist and the client was affected by this anxiety. He reported that throughout all the studies examined that fear, anger, and stress were all common within psychotherapists, and that therapist levels of fear are subjective, because they may report lower levels or higher levels of fear than what the situation presented. One of the studies examined in Harris's analysis of Pope and Tabachnick (1993, p. 146), in this article, they found that therapist and client gender had significant effects on the fear of whom the target of aggression would be. Male therapists reported being more worried about a potential attack on 1/3 party by the client. They also found that male clients created more fear of attack on the therapist than female clients (Pope \& Tabachnick, 1993, p. 146). This shows that the therapists' fears of a client need to be taken into account when debating therapeutic treatments and outcomes. One location in which there are a plethora of violent clients would be the correctional system. 


\section{Prison Settings}

There are few places where one can find more individuals with a violent history than in the US prison system. Most states have guidelines to rate the security levels and threat of violence each inmate can possess. Texas is just one state that has a rating system comparable to most other states within the US. In the Texas rating system, the prisoners are rated on a 1-5 scale with " 1 " being the lowest security level and " 5 " being the highest. To have the ranking of a Level 5 inmate, the inmate must have either assaulted two people within the past year or used a weapon on someone within the past year (Texas parole watch). They may also have been convicted for extortion or sexual abuse within the past two years. The final action that may land an inmate in Level 5 would be if they have escaped any correctional facility within the past five years. These are the requirements for being placed in a maximum security level within the state of Texas. The requirements to be placed at a Level 4 security are similar; the inmate must have had one disciplinary conviction for assaulting someone without using a weapon in the past year, escaped from prison, assaulted a prison staff member, or taken a hostage in the past 10 instead of five years, or have had two non-assaultive disciplinary convictions in the past six months. These patients are not as big of risks for violence as with the Level 5 security inmates. Level 3 security inmates are even a lower risk for violence but still have violent tendencies. These inmates that have assaulted fewer than three people within the past two years, have a clear conduct record for six months. The Level 3 inmate also must not have escaped from prison, assaulted prison staff, or taken a hostage within the past 10 years. The final qualifier to be a Level 3 inmate is that they have a prison sentence of more than 50 years and yet have to serve for a minimum required amount of time. The lowest level of prison that was examined would be those that contain a minimum of Level 3 security inmates, as that was the best way to assess clinician's responses to violent clients because they would be most likely to treat violent individuals on a more consistent basis.

\section{The Purpose of the Present Study}

This study looks at an area within the realm of psychology that has been severely understudied and is instrumental in examining the relationship between therapist and client; and the relevance of anxiety of violence on how the therapist uses self-involving statements. There are studies on the use of self-disclosure and more specifically self-involving statements, and there are also studies on the relevance of therapist anxiety. This study examined the relationship between them. Several hypotheses were tested:

(1) Therapists who deal with violent clients will use fewer negative self-involving statements;

(2) There will be no effect on positive self-involving statements between the correctional and non-correctional groups;

(3) Prison therapists who deal with violent clients will use fewer self-involving statements overall than non-correctional psychologists;

(4) Providers with higher self-reported anxiety will likewise report less frequent use of negative self-involving statements when compared to provider's reporting low levels of anxiety;

(5) There will be different motives for using self-involving statements between the correctional and non-correctional groups.

\section{Method}

\section{Participants}

This study used a clinical psychology, and the participants had work experience in multiple fields. This 
included 22 members with reported correctional experience, 62 reported working in a private practice, nine reported working in a community mental health center, 10 reported working in a private hospital, six reported working in a state/community hospital, 10 reported working in a university/college counseling center, three reported working in a school, and 25 reported working in a place not listed by the survey. These participants were part of a list of 1,764 participants selected from the APA (American Psychological Association) database yielding 147 participants, including 22 members of APA division 41 American Psychology-Law Society. The remaining were from APA division 12 Clinical Psychology. Of the total 144 participants, 93 were male and 51 were female. The average age of the therapists in these groups was 52.74 years old $(S D=10.72)$. The average experience that these professionals had was 23.27 years $(S D=11.20)$.

\section{Materials}

The CATS questionnaire as used and amended by Barnett and Lunnen (2008) was further modified to add questions involving client history of violence, and questions that ask about therapist anxiety (see Appendix A). This scale was found to have a Chronbach's alpha internal consistency score of 0.88 . The original questionnaire by Biegel (1994) had a Chronbach's alpha score of 0.87 and a test-retest reliability rating of 0.88 . The questionnaire asked frequencies of different possible statements that a psychotherapist may state to a client. There are two different types of self-involving statements tested, positive and negative. The positive statement is that a therapist expresses happiness with a client's progress, whereas the negative statement is that a therapist expresses displeasure of the client's progress/actions. These questions are rated on a 1-7 scale with " 1 " being that they have never used the given statement and with "7" being that they have used that statement in an extreme amount. Barnett and Lunnen (2008) added questions about the frequency of use of self-involving statements also with a 1-7 rating scale. Questions that were added to this measurement included if they have ever had to activate emergency response while seeing a patient due to risk of violence, how often they feel anxious when dealing with violent clients, percentage of clients that they see who are violent, what type of security level prison they work in, how often they have been threatened with physical harm by a client, and how often they have been physically assaulted by a client. These questions were added to respond to the second part of the hypothesis to determine the level of anxiety and if there were any correlations between the added questions which were compared to the total number of self-involving statements reported along with ratings from both the positive and negative groups.

\section{Procedure}

A link to the CATS questionnaire was placed on the Website to the correctional psychologist association - the International Association for Correctional and Forensic Psychology (This Website is http://www.aa4cfp.org/index.php?submenu=Home\&src=). This link was also sent with a brief paragraph explaining the study (see Appendix B) to 158 members (with correctional experience listed) of APA division 41 and 1,642 members of APA division 12. The correctional psychologists were informed that they could email the researcher with any questions.

\section{Results}

To test the hypothesis that therapists who deal with violent clients will use fewer negative self-involving statements due to fears of violence and that there will be no effect on positive self-involving statements within the two groups, a $t$-test was run. The negative statements show the correctional psychologists $(M=22.82 ; S D=$ 
6.54) actually used more negative self-involving statements than the non-correctional group $\operatorname{did}(M=20.52$; $S \mathrm{D}$ $=4.84), t_{(142)}=-1.94, p<0.05$. Thus, the hypothesis was not supported. To test the hypothesis that there will be no effect on positive self-involving statements within the two groups, a $t$-test was also run. The correctional group ( $M=29.68 ; S D=8.37$ ) used fewer positive self-involving statements than the non-correctional group did $(M=34.26 ; S D=6.44), t_{(142)}=2.92, p<0.05$. This hypothesis was also rejected. Overall the results indicate that correctional psychologists use more negative self-involving statements and fewer positive self-involving statements than populations of psychotherapists in other treatment areas.

To test the hypothesis that therapists who deal with violent clients will use fewer self-involving statements overall it was also tested with a $t$-test. This $t$-test compared answers with the question asking how often the therapist perceives their own level of self-involving statement use when compared to his or her colleagues. Confirming the hypothesis, the correctional group reported using fewer self-involving statements $(M=3.32$; $S D=1.89)$ when compared with non-correctional psychologists $(M=4.07 ; S D=1.25), t_{(142)}=2.37, p<0.05$. This suggests that overall correctional psychologists use fewer self-involving statements than psychologists in other clinical fields.

Table 1

Negative CATS Score as a Predictor of the Total Number of Emergency Situations

\begin{tabular}{lll}
\hline Predictor & $B$ & $S E-B$ \\
\hline Constant & -1.00 & 0.94 \\
Negative CATS score & $0.09^{*}$ & 0.04 \\
\hline
\end{tabular}

Note. ${ }^{*}$ denotes significance at 0.05 level.

The fourth hypothesis that correctional psychologists reporting higher levels of anxiety would also use fewer negative self-involving statements was tested by a correlation and a regression. The correlation demonstrates that there may be a relationship between how often a correctional therapist has to activate emergency response and the number of negative self-involving statements; this positive correlation $r_{(22)}=0.43$, $p<0.05$ points to a higher number of emergency situations with a higher use of negative self-involving statements. The regression analysis (see Table 1) suggests that the more emergency situations the clinician has, the more they will use negative self-involving statements. There is no significant relationship between the number of self-involving statements and reported anxiety levels.

To test the hypothesis that there will be different motives for using self-involving statements between the correctional and non-correctional groups, $t$-tests were tested by several independent samples. This was examined by looking at the motive questions asking how much of the decision to use self-involving statements was based on strengthening alliance, satisfaction with sharing/being open in the therapeutic relationship, convincing the client to relax, and compensating for a lack of openness within the clients within other relationships. The $t$-tests suggest that correctional psychologists $(M=3.18 ; S D=1.92)$ when compared to non-correctional psychologists $(M=4.83 ; S D=1.40)$, viewed strengthening alliance as less of a motive for using self-involving statements $t_{(142)}=4.79, p<0.001$. The correctional psychologists $(M=1.91 ; S D=1.30)$ when compared to non-correctional psychologists $(M=2.68$; $S D=1.30)$ also viewed satisfaction of openness within the therapeutic relationship as less of a motive for using self-involving statements $t_{(142)}=2.55, p<0.05$. The other two reasons of convincing the clients to relax and compensating for a lack of openness in other relationships produced non-significant results. This demonstrates that there are differences in motivation for 
using self-involving statements, with the non-correctional groups viewing being open in the therapeutic relationship and therapeutic alliance as being more of a motive for using self-involving statements.

\section{Discussion}

The first hypothesis that therapists who deal with violent clients will use fewer negative self-involving statements was not supported. Clinicians who worked in the correctional setting actually used more negative self-involving statements than clinicians in non-correctional settings. One possible explanation of this is that correctional psychologists are in the situation where they deal with individuals who do not function well in society, and that may give them a jaded opinion of their clients.

The second hypothesis that there will be no effect on positive self-involving statements between the correctional and non-correctional groups was also not supported. The correctional psychologists actually used fewer positive self-involving statements. This finding is consistent with research done by Harris (1999), showing that there are more different styles of treatment used for clients with violent tendencies than those who are treated without violent tendencies. This can expand on that assumption by adding that in terms of the use of self-involving statements, treating a violent client base may make a psychotherapist less likely to use a positive self-involving statement and more likely to use a negative self-involving statement.

The third hypothesis that correctional therapists who deal with violent clients will use fewer self-involving statements overall than non-correctional psychologist was accepted. This also shows differences in treatments when dealing with violent clients. This is relevant because it shows that groups who deal with more violent clients are willing less overall to give opinions on their clients' progression through treatment.

The fourth hypothesis that some providers with higher self-reported anxiety will likewise report less frequent use of negative self-involving statements when compared to provider's reporting low levels of anxiety was not supported. Due to the fact that individual anxiety was not significant, a comparison to the Harris's (1999) study can be made: A therapist's self-rated anxiety may not be the best measurement of therapist anxiety which is why the number of emergency situations is more focused on. The result that there is an increase in emergency situations seems to fall hand-in-hand with the study done by Dowd and Barato (1982), who found that clients tend to have more of an alliance with clinicians who use positive self-involving statements. This compares to the higher number of negative self-involving statements used when compared to the number of emergency situations per clinician. This suggests a correlation between higher amounts of negative self-involving statements and potential client violence.

The final hypothesis that there will be different motives for using self-involving statements between the correctional and non-correctional groups was also accepted. The present study suggests that types of and motives for the use of self-involving statements differ for clinicians who work in a more violent setting as opposed to everyday clinicians. This presents that clinicians with a less violent client base are more concerned about maintaining a healthy therapeutic alliance and making up for a lack of openness in other relationships in which the client may have than the group that deals with a more violent clientele.

\section{Suggestions for Future Research and Limitations}

With the results of the present study presenting such results, a few things could be done to further research. This topic would be to video record sessions of psychotherapists when they are dealing with violent and non-violent clients. This would allow the researcher to count the types of disclosure and the frequencies of 
disclosure with the same set of psychotherapists when dealing with violent and non-violent clients. After recording the video data, the psychotherapist and the client could complete various disclosure surveys to compare with the video data. The present study did offer some benefits that the suggested study would not be able to examine. Some of these benefits include examining the differences between different psychological occupations and the effects of occupation on the use of self-involving statements. This study is also more controlled than a video of different clients, in such a study as suggested no two clients would have the same issues and in some situations disclosure may be more appropriate than others.

As previously mentioned, out of the 1,764 names on the email list that were emailed, only 147 responded, which is roughly $8.33 \%$ of the selected participants. This presents two problems. The low percentage of responses suggests that this study may not have an accurate representation of the population groups. This can be explained by the difference that governs correctional staff in different states. In states like Ohio, researchers cannot use correctional staff or inmates without Department of Correction approval (Ohio DRC (Department of Rehabilitations and Corrections) resolution 06-RES-01, 2008). In other states, some psychological treatment style surveys to staff are more acceptable. Some of the correctional participants have recently stopped working in the prison which they are formerly employed at, but are still capable of completing the survey in a manner as to not present confounds. This means that they have been out of the correctional field for less than three years. The second problem is that there were only 22 participants in the correctional group, as previously stated that this puts the correctional staff on par with the numbers of the other participating groups.

Benefits of the present study include that differences in treatment by occupations were examined, the high response rate of the main focus group, and that the study was more controlled than video recording therapy sessions. In examining different occupations of psychotherapist's employment, this study was able to target a specific occupation (correctional psychotherapy) to best create a dichotomy between occupations that would regularly see violent clients and an occupation that would not. The high response rate of the main focus group (correctional psychotherapists) was beneficial to the study, because that group was asked the follow-up questions about anxiety with over $20 \%$. The other benefit of the study being more controlled comes from the nature of a survey based design. If therapist sessions were recorded, then there would be no way to create uniformity, because no two problems and no two clients are exactly the same. Overall the study was an efficient look at how violent clients can affect the use of self-involving statements. In the future, there should be more studies that look at how therapists react differently with different client types.

\section{References}

Andersen, B., \& Anderson, W. (1985). Client perceptions of counselors using positive and negative self-involving statements. Journal of Consulting Psychology, 32, 462-465.

Barnett, K., \& Lunnen K. M. (2008). The use of therapist self-involving statements with child and adult psychotherapy clients. New Wilmington, P. A.: Westminster College.

Biegel, A. E. (1994). Client perception of therapist self-disclosing and self-involving statements: An empirical method. San Diego, C. A.: California School of Professional Psychology.

Dowd, E. T., \& Barato, D. R. (1982). Differential effects of counselor self-disclosure, self-involving statements, and interpretation. Journal of Counseling Psychology, 29, 8-13.

Glanci, G., \& Saini M. A. (2005). An evidenced-based review of psychological treatments of anger and aggression. Brief Treatment and Crisis Intervention, 5(2), 229-249. doi: 10.1093/brief-treatment/mhi013

Guy, J. D., Brown, C. K., \& Poelstra, P. L. (1992). Safety concerns and protective measures used by psychotherapists. Professional Psychology: Research and Practice, 23(4), 21-42. 
Harris, A. (1999). Incidence and impacts of psychotherapists' feelings toward their clients: A review of the empirical literature. Counseling Psychology Quarterly, 12(4), 363-375. doi: 0951-5070/99/040363-13

Hudson-Allez, G. (2006). The stalking of psychotherapists by current or former clients: Beware of the insecurely attached! Psychodynamic Practice, 12(3), 249-260. doi: 10.1080/14753630600765550

Hunter, D. S. (1989). The use of physical restraint in managing out of control behavior in youth: A frontline perspective. Child and Youth Care Quarterly, 18, 141-155.

McCarthy, P. R., \& Betz, N. E. (1978). Differential effects of self-disclosing versus self-involving counselor statements. Journal of Counseling Psychology, 25, 251-256.

Ohio Department of Rehabilitations and Corrections, Research Division. (2008). Research approval process (06-RES-01). Columbus, O. H.: Ohio DRC.

Pope, K. S., \& Tabachnick, B. G. (1993). Therapists' anger, hate, fear, and sexual feelings: National survey of therapist responses, client characteristics, critical events, formal complaints, and training. Professional Psychology: Research and Practice, 24, 142-152. doi: 0735-7028/93

Reynolds, C. L., \& Fischer, C. H. (1983). Personal versus professional evaluations of self-disclosing and self-involving counselors. Journal of Counseling Psychology, 30(3), 451-454. doi: 10.1037/0022-0167.30.3.451

Sharkin, B. S., \& Gelso, C. J. (1993). The influence of counselor trainee anger-proneness and anger discomfort on reactions to an angry client. Journal of Counseling \& Development, 71(5), 483-487. doi:10.1002/j.1556-6676.1993.tb02229.x

Texas Parole Watch Explanation of Custody Levels. (2007, September 5). Retrieved from http://www.texasparolewatch.info /section_three/custody.aspx

\section{Appendix}

Appendix A

\section{Self-involving Survey}

The questions on this survey are about the self-involving statements therapists sometimes make. Self-involving statements, as opposed to other forms of self-disclosure, are statements that express the therapist's feelings/attitudes towards the client while in session. Please answer the following questions with regard to your own use of self-involving statements with your typical clients. We are particularly interested in your thoughts as they relate to your clients who have a history of violence.

Questions marked with a * are required.

*1. What is your age?

*2. What is your gender?

G Male

C Female

*3. What is your predominant theoretical orientation?

C. Psychodynamic/Existential

C. Behavioral

C. Cognitive

C Humanistic

*4. Years of clinical experience?

*5. What is your primary treatment population?

[. Children (5-7)

C Children (8-12)

C. Youth (13-17)

C Adults (non-geriatric) 
C. Adults (geriatric)

*6. In what type of correctional institution are you employed?

G. Minimum security

C Medium security

C. Maximum security

G Other

*7. What is your predominant mode of treatment?

C INdividual

C. Group

C Community/prevention

*8. On average, how many hours do you spend per week in clinical practice?

*9. How often do you feel anxious/fearful when working with clients with a history of violence?

C. Never

C. Rairly

Occasionally

C. Frequently

C. Almost Always

*10. How often have you been threatened with physical harm by a client?

C. Never

C 1 time

C.3 times

C.6 times

[7+ times

*11. Have you ever had to activate emergency response while seeing a patient due to risk for violence?

[. Yes

C No

*12. If yes, how frequently?

C 1 time

C.3 times

[. 4-6 times

C 7 or more times

*13. How many times, if any, have you actually been assaulted by a client?

C Never

C 1 time

[. 2-3 times

[4-6 times

[. $7+$ times

*14. Compared to your colleagues, how often do you tend to use self-involving statements with your clients?

C 1 = much less frequently

E 2 


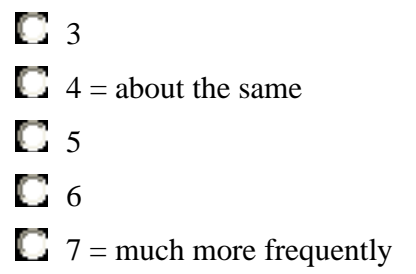

*15. How much of your decision to use a self-involving statement is based on a goal to strengthen therapeutic alliance?

C Not at all

C Slightly

C Somewhat

C Moderately

C Considerably

C Very much

C Extremely

*16. How much of your decision to use a self-involving statement is based on your own satisfaction with sharing/being open?
C Not at all
C Slightly
C Somewhat
C Moderately
C. Considerably
C Very much
C Extremely

*17. How much of your decision to use a self-involving statement is based on a goal to help the client relax?

C Not at all

C Slightly

C Somewhat

C Moderately

C Considerably

C Very much

C Extremely

*18. How much of your decision to use a self-involving statement is based on a goal to compensate for a lack of openness in the client's other relationships?
C Not at all
C Slightly
C Somewhat
C Moderately
C Considerably
C Very much
C Extremely

*19. I have expressed feelings of relief to a client's statements or behaviors.

C Never 

C Rarely
C Sometimes
C Several times
C Frequently

*20. I have told a client that I have felt proud of them.

C Never

C Rarely

C Sometimes

C Several times

C Frequently

*21. I have expressed feelings of surprise in response to a client's statements or behaviors.

C Never

C Rarely

C Sometimes

C Several times

C Frequently

*22. I have expressed feelings of admiration towards a client.

C Never

C Rarely

C Sometimes

C Several times

C Frequently

*23. I have told a client that I have respect for them.

C Never

C Rarely

C Sometimes

C Several times

C Frequently

*24. I have told a client that I felt delighted by their statements or behaviors.

C Never

C Rarely

C Sometimes

C Several times

C Frequently

*25. I have expressed feelings of compassion towards a client.

C Never

C Rarely

C Sometimes

C Several times

C Frequently

*26. I have responded to a client’s statements or behaviors by expressing curiosity. 

C Never
C Rarely
C Sometimes
C Several times
C Frequently

*27. I have expressed feelings of optimism towards a client.

C Never

C Rarely

C Sometimes

C Several times

C Frequently

*28. I have expressed feelings of appreciation to a client's statements or behaviors.

C Never

C Rarely

C Sometimes

$\boldsymbol{C}$ Several times

C Frequently

*29. I have expressed feelings of sadness to a client's statements or behaviors.

C Never

C Rarely

C Sometimes

C Several times

C Frequently

*30. I have told a client I have felt frustrated with them.

C Never

C Rarely

C Sometimes

$\mathbb{C}$ Several times

C Frequently

*31. I have expressed feelings of discouragement to a client's statements or behaviors.

C Never

C Rarely

C Sometimes

C Several times

C Frequently

*32. I have told a client that I have felt impatient with them.

C Never

C Rarely

C Sometimes

C Several times

C Frequently 
*33. I have expressed feelings of being alarmed by a client's statements or behaviors.

C. Never

C. Rarely

C Sometimes

C. Several times

C Frequently

*34. I have expressed feelings of boredom towards a client.

C. Never

C. Rarely

C Sometimes

C. Several times

C Frequently

*35. I have expressed feelings of suspiciousness to a client's statements or behaviors.

C. Never

C. Rarely

C Sometimes

C. Several times

C Frequently

*36. I have told a client that I felt worried about them.

C. Never

C. Rarely

E Sometimes

C. Several times

C Frequently

*37. I have expressed feelings about being frightened by a client's statements or behaviors.

[. Never

C. Rarely

C Sometimes

E Several times

C Frequently

*38. I have expressed anxiety to a client's statement or behaviors.

C Never

C. Rarely

C Sometimes

E Several times

C Frequently 


\section{Appendix B}

Dear Dr.

Hello, my name is Kirk M. Lunnen. I am an associate professor of psychology at Westminster College in New Wilmington PA. My research team is conducting a study of therapist self-disclosure practices with a particular focus on providers with current or previous experience in correctional settings. I have attached a link to a brief survey. We would greatly appreciate a few minutes (about 5-10 minutes) of your time to complete our survey. Our hope is to develop a greater understanding of therapists attitudes concerning therapist self-disclosure when working in a correctional environment.

All responses are completely anonymous. If you have any questions or would later like the results of this study, do not hesitate to contact me. Many thanks for your help.

The survey is available at: http://unix4.psych.westminster.edu/phpESP/public/survey.php?name=Self_involving_test_Prison

Kirk M. Lunnen, Ph.D.

Department of Psychology

Westminster College

New Wilmington, PA 16172

Tel: (724) 946-7203

E-mail: lunnenkm@westminster.edu 